\title{
Energy intensitites, EROI (energy returned on invested), for electric energy sources
}

\author{
D. Weissbach, F. Herrmann, G. Ruprecht and A. Huke \\ Institut für Festkörper-Kernphysik gGmbH - Leistikowstr. 2, 14050 Berlin, Germany
}

\section{K. Czerski}

Institut für Festkörper-Kernphysik gGmbH - Leistikowstr. 2, 14050 Berlin, Germany

Instytut Fizyki, Wydziat Matematyczno-Fizyczny, Uniwersytet Szczeciński

ul. Wielkopolska 15, 70-451, Szczecin, Poland

\section{S. Gottlieb}

Institut für Festkörper-Kernphysik gGmbH - Leistikowstr. 2, 14050 Berlin, Germany

Fachhochschule Dortmund - Sonnenstraße 96, 44139 Dortmund, Germany

\author{
A. Hussein \\ Department of Physics, University of Northern British Columbia \\ 3333 University Way, Prince George, BC, V6P 3S6, Canada
}

\begin{abstract}
Summary. - The EROI (Energy Returned On Invested) has been calculated for various systems, especially power plants, applying several different methodologies. This quantity is often used as an argument in discussions about economics to emphasize the performance of a certain technology. Contrary to that, the publications often answer different questions, e.g. analyses of emissions or material flows, rendering the EROI unphysical. The present work introduces a physical approach based on exergy flows for EROI calculation to pinpoint and compare the efficiency of different power plant technologies.
\end{abstract}

\section{1. - Introduction}

The economic strength of a society depends heavily (or even relies predominantly) on two factors: how efficiently it can provide valuable (or better: usable) work - hereafter 
called exergy - and how proficient it is at using it for the production of goods and services. Measuring this efficiency by investigating the flows of money, goods and services is a complex task, due to unreproducible and arbitrary changes caused by societal processes such as political decisions. Therefore, it is expedient to introduce quantities that are invariant against these issues - physical quantities.

Note that in the present work, the term "society" is defined as: A large group of humans living together over several generations, while operating an economic and industrial system to provide goods and services for themselves and possibly others. Thus, all modern nations fulfill our definition of society. A small set of millionaires living on an island and importing all necessities from the outside is not a society meeting our criteria.

While it is difficult (yet possible) to investigate the physical benefit of goods and services, evaluating a society's capability of gaining usable work is far more intuitive. In order to provide exergy, a certain amount of exergy has to be invested. A motor which delivers mechanical work or electricity has to be built first, and the motor factory will require some work and electricity to do so. Thus, one crucial question arises: Which fraction of the available usable work (or exergy) has to be invested to provide it? The answer lies in a single quantity: the EROI, Energy Returned On Invested, which has been determined for several technologies used to deliver exergy.

These systems are heat engines, because only they are able to provide more exergy than they consume. Keep in mind that exergy is different from energy in general, as the latter encompasses both diffuse (heat, noise) and ordered energy (electricity, mechanical work, lasers, etc.), while the term exergy refers exclusively to the second type. For a technological society, the most important heat engines are vehicle motors (piston and turbine/jet engines) on the one hand and electrical power plants on the other: the roles of both kinds are highly intertwined, so that the failure of one would cause immediate social chaos. As automation progresses with computers and robots taking over increasingly large parts of the economy, the importance of electrical power plants will grow proportionally.

In fact, heat engines can be seen as economic amplifiers, with the EROI, or $R$ for short, as the amplification factor. Man provides 1 unit of usable work - and technology amplifies it $R$-fold by delivering $R$ units. Using a more vivid metaphor, the EROI tells us how much work machines can do for us. Thus, the historical change of $R$ of society's power generation is the most important proxy for industrial progress and wealth evolution, hovering around 1 for most of human history, until industrialization - the large-scale application of heat engines - began boosting it upwards starting in the late 18th century, reaching values of multiples of 10 for OECD-like societies of today.

The term EROI has been used in many ways to answer questions different from the one above. It is often applied inconsistently, inhibiting the comparison of the economic efficiency of power plant technologies. This lecture, based on the respective publication [1] of one of the lecture authors and his colleagues, introduces a physical approach for obtaining the EROI that not only potentially eliminates these flaws but may also provide answers to the question of how certain types of power plants affect the economic efficiency of a society. 


\section{2. - Description}

It is crucial to treat the EROI (or $R$ just for practical mathematical notation) as a physical quantity. From an industrial standpoint, mutability is the most important attribute of a certain form of energy, allowing its adaptation to any purpose. Disordered energy (also called anergy, that is, heat, noise or diffuse radiation) can only partially be converted to exergy, while exergy can always be converted completely to anergy. This is what gives exergy provided by heat engines its central importance: It is versatile, it can be transformed and transported over great distances as electrical current. Heat has its uses too, e.g. enabling proper factory operation, but it is not able to do any useful work alone. In order to create goods and services, coherent, ordered energy is needed - exergy. The maximum amount of exergy that can be extracted in a closed system is limited, but from the point of view of a society, it can be generated when and where needed, provided that fuel supply is not cut off.

Speaking in terms of statistical physics, only the application of exergy is able to reduce the entropy of an (open) system. For a more thorough explanation of these terms, the reader is referred to introductory literature on engineering, thermodynamics and statistical physics, thus in this work extensive explanations will be skipped in order to avoid disturbing the reading flow.

The EROI $R$ of a power plant can be calculated from the following simple equation:

$$
R:=\frac{E_{\mathrm{out}}}{E_{\mathrm{in}}} .
$$

with the total electricity output $E_{\text {out }}$ and the cumulative demand or input $E_{\text {in }}$, which both, ideally, should be exergies.

The electricity output is, by definition, pure exergy, but the input has to be carefully evaluated, since the data often contains no information about the energy type, making it difficult to extract or convert to exergy. Nevertheless, it is consistent to exclusively use the same pure physical quantity, which can be uniformly applied to all thermodynamic machines, both for output and input.

The methodological conditions for obtaining the output and the input should further ensure that all relevant inputs (fuel extraction, construction, buffering, maintenance, etc.) are considered to achieve comparability (see below).

\section{3. - Methodology}

Strict exergy usage only: In order to maintain comparability, at least the output of the power plant should not contain any non-exergetical parts like waste heat, since some plant types (wind, photovoltaics) provide electricity exclusively. As mentioned above, only exergy (here: electricity) is usable for industrial processes, e.g. construction and operation of power plants, while heat is far less versatile. Most of all, its thermodynamic quality depends on its temperature - lower temperatures meaning lower quality — which is not uniform among power plant technologies. 
No quality weighting of output: Several works present an equivalent of the electricity output of renewables, but not the output itself, to express the amount of fuel (coal, natural gas, ... ) not used in a fossil-fueled plant and thus saved. The "EROI" obtained by this approach answers a different question: how much more fuel can be saved by further expanding a certain renewable technology. This addresses resource stockpile problems, but has little to do with the topic at hand; therefore, the "EROI" derived in these works is not an EROI from the standpoint of physics. For the physical approach, this issue is obviously completely irrelevant, thus only the unweighted electricity output must be considered.

No quality weighting of input: As mentioned above, input data often contain several energy fractions of different quality. If possible, only the unweighted amount of exergy used should be considered. In contrast, in most works, all mechanical work or electricity is converted to its primary energy equivalent by multiplying it with the inverse of the mean thermal efficiency of all power plants operated by the society. This is quite arbitrary, since the turbine efficiency might change and the conversion factor rather represents a market value difference. Low-quality heat alone is not sufficient to build and operate power plants, this renders the weighting approach unphysical.

Nevertheless, in the author's work, gathering sufficient data to use pure exergy inputs could not be achieved either. Thus, an unweighted addition of different energy types as well as fuel energy inventories was considered the best practical approach. This immediately causes comparability issues, since data of power technologies show different fractions of exergy usage. To mitigate this problem, all data and the calculations were collected in a table open to the public. See [2].

Considering input as-is: Some works analyzing exergy flows only use theoretical values derived from thermodynamics and chemistry, e.g. work amounts and chemical processes without friction or losses, assuming an efficiency of $100 \%$. That does not describe the actual economics of the society, but reflects the theoretical limits of a given technology which cannot be exploited due to physical reasons. Instead, the real used inputs are considered in this analysis.

Discarding fuel energy content: Coal, uranium or any other fuel can be considered a "thermodynamic black box", in the sense that its heat content is in itself not relevant for the society using it - only the usable work extracted from it is relevant for this approach. Thus, the exergy expended to mine fuel, and that gained by burning it and running the combustion heat through a thermodynamic engine enter our calculations, not the raw energy content of the fuel itself. Putting it more clearly: Humans never injected the primary energy content into fossil fuel stockpiles, they were created and made available by natural processes. Contrary to this, many analyses, e.g. Raugei et al., include the fuel's calorific value in the input, which thus becomes dominant for fuel-consuming power plants. This inherently causes the EROI to be roughly equal to the thermal efficiency of the plant, instead of signifying exergy amplification.

Usage of physical inputs only: As shown above, for the purposes of this analysis, only exergy flows should be considered. This is done by adding up all such relevant inputs from all relevant fields and processes, which is also known as a bottom-up analysis. 
So-called top-down investigations were performed in other works, where non-physical efforts like money transfer or labor costs are converted into energy. For instance, this is often done by linking the energy consumption to the gross-domestic product of a country. Since the conversion ratio is highly volatile and strongly depends on the location, it is hardly possibly to treat this as a physical quantity in the strict sense. Further details are mentioned in sect. $\mathbf{6}$.

Treat recycling as it is: In many works, recycling has been taken into account by completely subtracting material inventories which could theoretically be reused, assuming they will be fully recovered during the operation of the plant. In reality, this is not the case. Materials are only partially recycled - processing them to avoid downcycling consumes energy. Also, the recycling procedure does not necessarily take place during decommission: should we link the recycled material to the present power plant or a future one? To be consistent in this issue, our analysis uses the present material inventories which already include recycled fractions while omitting such assumptions as mentioned above.

\section{4. - System borders and auxiliary facilities}

As stated above, all relevant inputs have to be included, even, for example, the facilities that produce machines which are then used to extract fuel. To be more precise, the system has to be enlarged to encompass the infrastructure that created these facilities and so on. Obviously, this gets very complex when delving deeper into the infrastructure and it is necessary to perform an appropriate analysis truncation: system borders need to be defined. To this end, the following condition is used: The contribution of the remaining infrastructure outside of the limits should be lower than the error of the entire input estimate. Thus, auxiliary facilities such as storage units, mining sites as well as material inventory and exergy flows regarding the power plant are included. All infrastructure outside, such as production of mining machinery or lubricants, contributes negligibly and need not be taken into account.

Figure 1 shows a schematic of the industrial infrastructure around a power plant.

All input sources which fulfill the aforementioned conditions are shown. They are located outside the analyzed system, the power generating system including auxiliary facilities, as defined by the system borders. Note that some energy flows enter the system from the outside while others occur within it (e.g. electricity produced at the power plant to directly supply the plant's auxiliary systems). In the latter case, the demand is subtracted from the output instead of adding it to the input, as it remains within the analyzed system - it is not coming from the (consuming) society outside and therefore, from the "investor's point of view", it can be seen as internal friction.

A power plant not only has to produce electricity, but must also ensure that its output satisfies society's demand, so unpredictable volatility has to be eliminated via buffering (e.g. for wind farms). The following assumptions are made in this analysis:

- The output (as well as society's demand) is treated as constant base load. Maintenance outages of all power plants are mostly predictable and therefore neglected. 


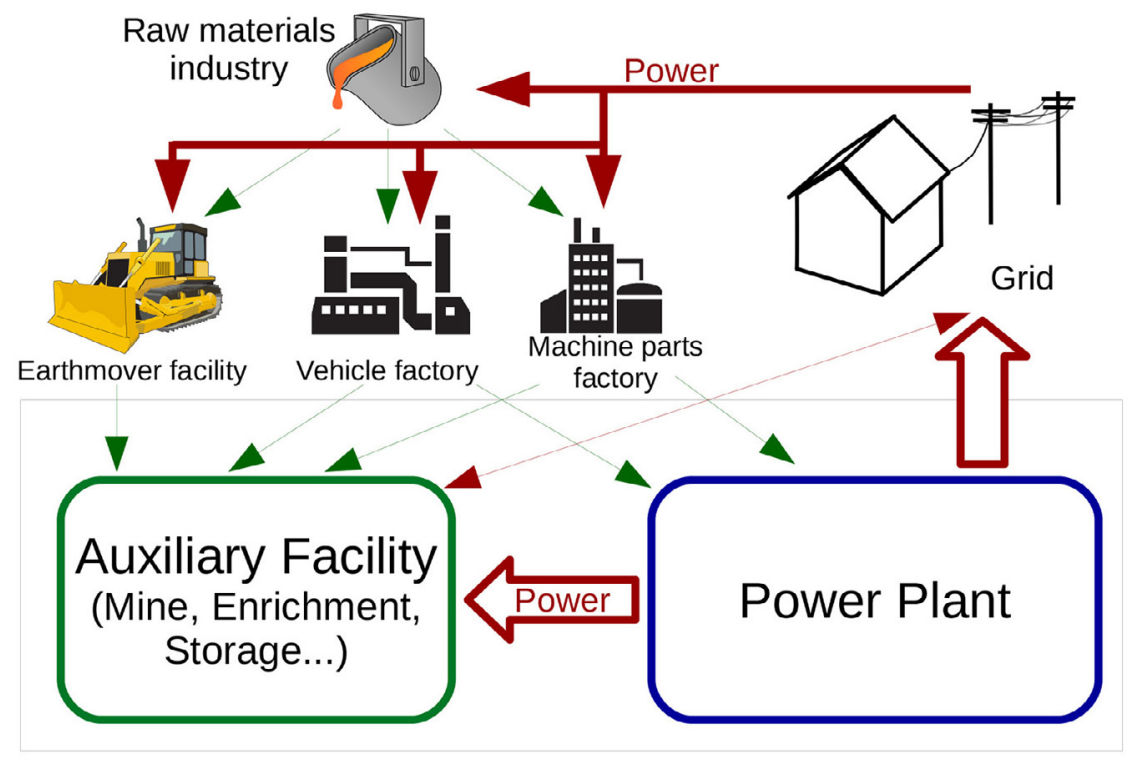

Fig. 1. - System borders for a typical power plant. Whether electricity for a auxiliary facility like a coal mine is taken from the grid (thin red line) or from the power plant does make a difference. Note that if the auxiliary facility is an energy buffer, it also feeds back to the grid.

This issue is already taken into account by the average annual load that technically can be achieved.

- The actual power technology has to fully supply the society by itself. Combinations with other technology mask the characteristics of the analyzed system and hampers comparability.

- The output has to follow load, not vice versa, as often proposed by advocates of smart grids. To this end, buffering for volatile techniques like wind and solar are required - though not for fueled plants as their fuel acts as the storage. Instead, fueled plants need mines or wells as an auxiliary facility, see fig. 1.

The buffering efforts can be estimated by using the data from Popp [3] and the calculation is explained in the authors' publication [1]. This intuitive approach — including buffering for volatile technologies instead of fuel for fueled plants - has so far never been used in any EROI works known to the authors today. Of course, one might argue that the society's demand is never base-load-like so non-volatile power plants have to adjust for that, but these predictable changes can be managed through optimized operation of such plants, not significantly reducing their EROI. Only for niche applications like air condition during the day in hot regions, unbuffered solar power is suitable, but this is by far not a full-supply scenario. 


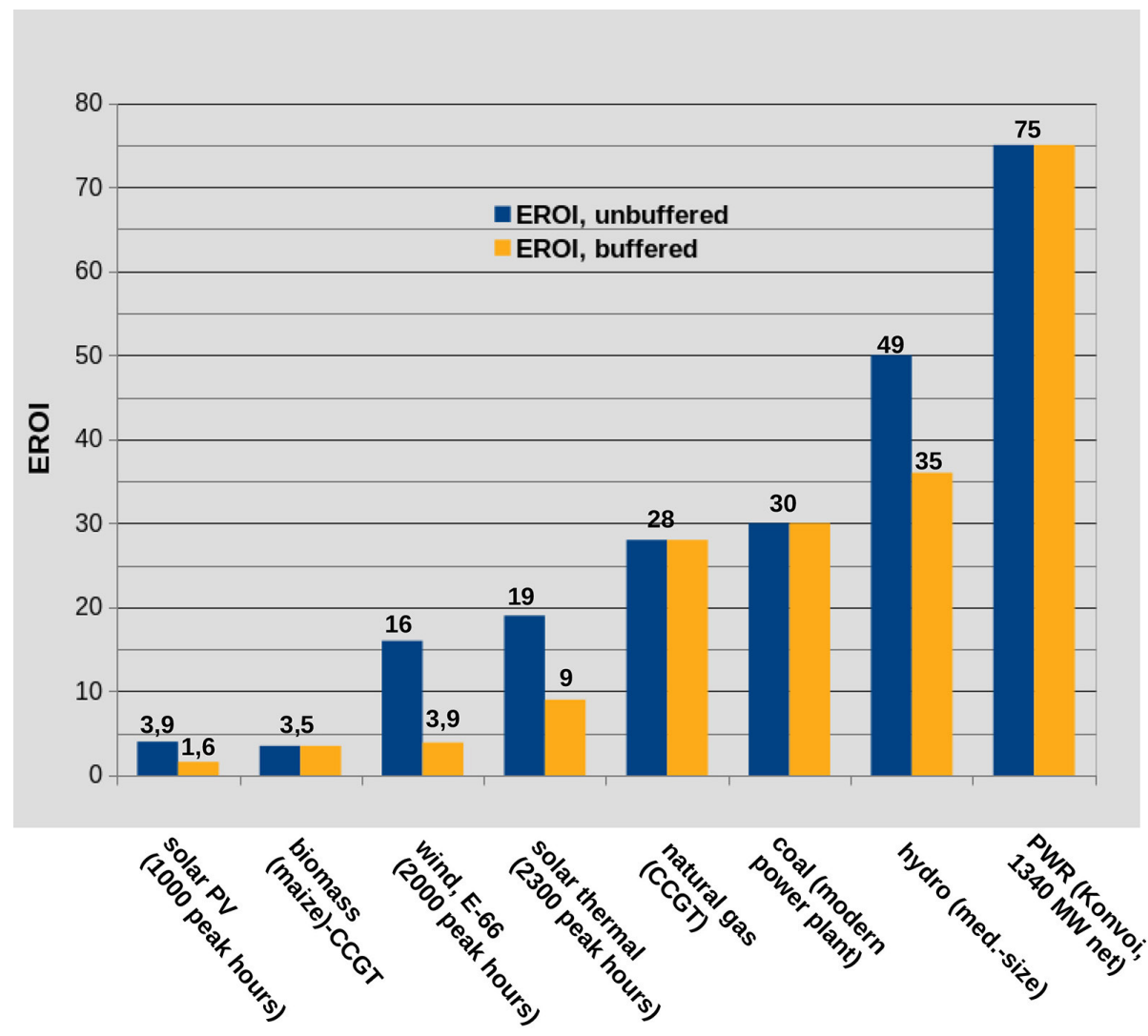

Fig. 2. - EROIs of several electricity generation technologies.

\section{5. - Results and nuclear power as example}

The diagram in fig. 2, taken from the authors' publication [1], shows the EROI of some power plant technologies. The respective parameters, also mentioned in this publication, are adapted to German conditions.

Unbuffered EROIs for the volatile technologies (solar, wind, etc.) are shown only for completeness' sake - electricity cannot be stored without converting it to some form of potential energy first, and as the EROI by definition compares exergies, unbuffered EROIs do not exist for fluctuating sources in a strict sense. Additional buffering efforts for all technologies due to load-following are very small compared to compensation for volatility, so they are neglected here.

The economies of all OECD nations rest on fossil burning. This means that in order to achieve what is perceived as prosperity in the 21st century, EROIs around 30 or larger are needed. More generally speaking, the EROI of non-mechanized human work (a singledigit value close to 1) should be exceeded by at least one order of magnitude, putting 
TABLE I. - EROIs and key figures of the reference nuclear power plant (100\% centrifuge enrichment in brackets), based on the authors' publication [1]. The values outside the brackets (before year 2000) correspond to a composition of $83 \%$ centrifuge and $17 \%$ diffusion enrichment where the latter consumes almost 50 times more electricity than the former.

\begin{tabular}{|c|c|}
\hline Installed capacity (net) & $1340 \mathrm{MW}$ \\
\hline Full-load hours & 8000 \\
\hline Lifetime & $60 \mathrm{a}$ \\
\hline Output & 2315000 TJ \\
\hline Construction energy demand & $4050 \mathrm{TJ}$, thereof $35 \%$ electrical \\
\hline Decommissioning energy demand & $1150 \mathrm{TJ}$, thereof $40 \%$ electrical \\
\hline Maintenance energy demand & $6900 \mathrm{TJ}$, thereof $68 \%$ electrical \\
\hline Fuel extraction & $4200 \mathrm{TJ}$, thereof $50 \%$ electrical \\
\hline Fuel element fabrication, conversion & $2480 \mathrm{TJ}$, thereof $15 \%$ electrical \\
\hline Fuel enrichment & $10740 \mathrm{TJ}$ ( $1560 \mathrm{TJ})$, thereof $100 \%$ electrical \\
\hline Fuel disposal & $1380 \mathrm{TJ}$, thereof $13 \%$ electrical \\
\hline Sum energy demand & $\begin{array}{l}30900 \mathrm{TJ}(21750 \mathrm{TJ}) \text {, thereof } 60 \%(50 \%) \\
\text { electrical }\end{array}$ \\
\hline EROI & $75(105)$ \\
\hline
\end{tabular}

the economic threshold at a value of around 10. Physically, this means that low-EROI technologies cannot stand on their own without hampering the present economic wealth in industrialized countries: they would collapse quickly if they had to deliver the energy needed for their own construction, maintenance and decommission. Current endeavors in (even unbuffered) solar and wind are critically dependent on fossil or nuclear power driving the production of units.

It is obvious that volatile technologies or power plants with low power density show the smallest EROI values. Wind energy (unbuffered) achieves relatively high values due to the mostly cheap (in terms of energy) materials, but its high fluctuations need substantial buffering. Coal and Combined-Cycle Gas Turbine (CCGT) show similar EROI values, but CCGT inputs are dominated to a far higher degree by fuel extraction expenses when compared with coal, while extraction and enrichment are the main input contributors of nuclear, see table I.

When looking at the distribution of monetary costs, remarkable differences to the energy input fractions can be observed. For instance, coal extraction dominates the monetary as well as the energy investment, but for nuclear power, fuel extraction plus enrichment absorbs the largest share of energy input, while costs are dominated by plant construction. This demonstrates the necessity of evaluating the physical economic efficiency of a given power plant — coal seems to be managed quite efficiently, but the 
technological potential of the Light Water Reactor (LWR) is not fully exhausted, as the electricity costs show: they are similar for coal and nuclear power plants despite the substantial EROI difference. In fact, the theoretical potential of nuclear power in general is exploited to a far smaller degree than that of all other existing technologies.

The caloric value of actinides is about 3 million times higher than that of chemical fuels. Even when accounting for the 1000 (mines today) to 30000 (mean concentration in Earth's crust) times lower abundance in nature, far higher EROIs than those of currenttechnology plants, surpassing fossil plants by only a factor of 2.5, should be achievable. LWRs are not capable of unleashing the full potential of the Strong Force. This is caused in part by their very low fuel usage (less than 1\%), as well as, relative to the amount, high fuel processing demand. Additionally, LWRs are complex facilities. Improving these flaws, together with a better economic exploitation of the technology, would enable nuclear to boost society to a completely new level of energy flux densities and productivity.

\section{6. - Comparison with other results}

The following example, shown in table II, demonstrates how the same EROI for solar photovoltaics can be obtained using different methodologies — see the analysis of Prieto [4] as compared to the present work. Detailed numbers can be found in his book [5]. The main difference is the extensive usage of additional top-down parts by Prieto vs. the higher buffering fraction assumed by Weißbach et al. - Prieto included lower backup efforts provided by the surrounding power plants.

Prieto used to operate large photovoltaic field installations in Spain, collecting extensive economic data on the whole production infrastructure and gaining comprehensive maintenance experience. His absolute input energy values for the modules are roughly the same as the ones from the author, the relative difference being caused simply by the different plant load. Comparing the output data of Prieto and Weißbach in their respective works shows that the electric efficiency as well as the output reduction due to weather, inverter efficiency etc., or the so-called performance factor is roughly the same. All these amounts are real used energies, but Prieto adds additional money-equivalent energy inputs.

About $60 \%$ of the entire input calculated by Prieto is derived from monetary costs and further $10 \%$ are calculated from the primary energy consumption per capita for the whole labor involved. This methodology shows some serious issues when calculating the EROI as a physical quantity. While it is accepted that money and labor are important proxys for evaluating the economic efficiency of power plant technologies, it heavily masks its physical efficiency in several ways.

First, the energy-to-money ratio as well as the per capita primary energy consumption depend heavily on the country the analyzed plant is located in or on societal processes in the said country. Although the plant itself is physically the same no matter which location is considered, the physical efficiency, the EROI, changes significantly - a gas turbine in a less developed country would have a higher EROI than in an industrialized country. This is caused simply by the different living standards, which brings us to the 
TABLE II. - Values are given as fractions from the output, so their sum corresponds to the energy intensity $1 / R$.

\begin{tabular}{|c|c|c|c|}
\hline & $\begin{array}{l}\text { Prieto } \\
(2016)\end{array}$ & $\begin{array}{l}\text { Weißbach } \\
(2013)\end{array}$ & Comments \\
\hline Manufacturing & $12 \%$ & $21 \%$ & $\begin{array}{l}\text { difference corresponds to } \\
\text { insolation }\end{array}$ \\
\hline Installation, transports & $3.5 \%$ & $4.1 \%$ & Prieto: mostly top-down \\
\hline Backup fraction & $4 \%$ & $18 \%$ & $\begin{array}{l}\text { Prieto: top-down (CCGT } \\
\text { backup), Weißbach: Pump } \\
\text { hydro }\end{array}$ \\
\hline direct/indirect labor & $5.5 \%$ & $0 \%$ & $\begin{array}{l}\text { Prieto: } 90 \mathrm{MWh} \text { /year per } \\
\text { worker (primary energy) }\end{array}$ \\
\hline Operation/maintenance & $7.7 \%$ & $0 \%$ & $\begin{array}{l}\text { Prieto: top-down, } \\
\text { Weißbach: no energy } \\
\text { data known }\end{array}$ \\
\hline $\begin{array}{l}\text { surveillance, taxes, } \\
\text { fees, insurance, PR }\end{array}$ & $3.5 \%$ & $0 \%$ & $\begin{array}{l}\text { Prieto: top-down, money- } \\
\text { to-energy conversion } \\
2 \mathrm{kWh} / \text { euro }\end{array}$ \\
\hline $\begin{array}{l}\text { losses, production } \\
\text { overcapacities, grid } \\
\text { fraction }\end{array}$ & $\begin{array}{l}8 \% \\
(3.5 \% \\
\text { grid-related })\end{array}$ & $\begin{array}{l}0.8 \% \\
\text { (grid } \\
\text { connection } \\
\text { only) }\end{array}$ & $\begin{array}{l}\text { Prieto: top-down, fraction } \\
\text { of whole grid included }\end{array}$ \\
\hline EROI, buffered & $2-3$ & 2.3 & $\begin{array}{l}\text { Poly-Si, field, Pietro: } \\
\text { 1730, Weißbach: } 1000 \\
\text { full-load hours per year }\end{array}$ \\
\hline
\end{tabular}

second issue. The living standards, which are now included in the input, are the result of the society's economic efficiency, which itself, at first, should be a consequence of the EROI. Such feedback loops in the EROI calculations lower the EROI for power plant technologies with high monetary flows (e.g. nuclear, see sect. 5) or which necessitate a lot of labor (e.g. solar, see above), considerably obfuscating the achievable physical efficiency and hampering comparability.

For this reason, such efforts are not taken into account in the present work. The solar PV system analyzed by Prieto thus would have an EROI of about 4 when assuming reasonable buffering. This value shows the maximum physical efficiency of the actual system with the available technology and a certain insolation, invariant against societal processes. Regarding other analyses, the manifold methodologies applied may cause EROI variations by a factor of 50 - mainly due to input or output weighting, top-down calculations and outdated numbers, best seen in the analysis of nuclear power plants by Leeuwen et al. [6], with an EROI of roughly 2. 
Prieto's analysis show a similar EROI value for solar PV compared to the work by Weißbach et al. indicating the systems having a similar economic performance. Nevertheless, the main difference is the methodology — Prieto used energy inputs derived from monetary flows, rendering the EROI more unphysical, making it not invariant against non-physical quantities thus less comparable to other technologies. While it is easily possible to adjust for the different insolation (1750 and 1000 full-load hours annually in Spain and Germany, respectively) it is not possible to compare both PV systems because of arbitrary societal influence - the administrative and salary situation in Spain differs from that in Germany. The results just seem to be accidentally identical because there are several derivations in both directions.

\section{7. - Conclusion}

In the large field of life cycle analysis, until now attention focused mostly on emissions, material and energy flows and also monetary quantities, answering questions about economy, environmental impacts and resource consumption. When used, the EROI was usually not treated as a physical quantity, but rather as a proxy for these issues. Thus, its value for a given system varied substantially from one publication to the other - it is actually hard to find two publications with the same result. The methodologies applied were neither consistent nor uniform among each other, and societal properties (e.g. monetary flows) were often included indirectly in the analysis. This approach not only disguises economical properties of the system as physical quantities (i.e. especially its physical efficiency), but also inhibits comparability among analyzed systems.

The present work introduces a consistent method for calculating the EROI, emphasizing the inherent physical characteristics of power plants, which are among the most important infrastructure elements for technologically advanced societies. It was found that only real used exergy flows should be included in the input and output, but the lack of sufficient data makes it hard to do so. Nevertheless, with the raw data presented in a transparent way, EROI values for several power plant technologies have been calculated using a consistent methodology. Comparing them gives strong clues to their benefit for industrialized societies. It comes as no surprise that technologies with the highest power and energy density as well as the lowest volatility show the highest EROI values.

There are numerous parameters which can also be discussed, such as fuel resources, "death toll" or land use; but these are independent problems and should be treated separately (though fuel scarcity has a certain influence on the EROI). Combining all these aspects will hopefully allow for a sound discussion about present and future energy supply. 


\section{REFERENCES}

[1] Weissbach D., Ruprecht G., Huke A., Czerski K., Gottlieb S. and A. Hussein, Energy, 52 (2013) 210, http://dx.doi.org/10.1016/j.energy.2013.01.029.

[2] Google+ table this work is based on: https://docs.google.com/spreadsheets/d/ 11BK3pntKdd3bo8oAAvjnpQvYaLZp1G-ieuS5GA5NGV4.

[3] Popp M., Speicherbedarf bei einer Stromversorgung mit erneuerbaren Energien (Springer, Berlin) 2010, pp. 135-137, http://www.springer.com/engineering/energy+technology/ book/978-3-642-01926-5.

[4] P. Prieto, Science 85 Energy, Course 3 at Ecole de Physique des Houches, presentation, Les Houches 2016, http://science-and-energy.org/wp-content/uploads/2016/03/ 20160307-Des-Houches-Case-Study-for-Solar-PV.pdf.

[5] Pedro A. Prieto and Charles Hall, Spain's Photovoltaic Revolution (Springer, New York) 2013, http://www. springer.com/gb/book/9781441994363.

[6] Storm van Leeuwen J. W., Nuclear power - the energy balance, website, Part D, Ceedata Consultancy 2007. Visited 2017/07/18, http://www. stormsmith.nl/np-ebalance.html. 